\title{
Basic Elastic Properties Predictions of Cubic Cerium Oxide Using First-Principles Methods
}

\author{
Jon C. Goldsby \\ Glenn Research Center, National Aeronautics and Space Administration, 21000 Brookpark Road, \\ Cleveland, $\mathrm{OH} 44135$, USA \\ Correspondence should be addressed to Jon C. Goldsby; jon.c.goldsby@nasa.gov \\ Received 29 May 2012; Accepted 11 June 2012 \\ Academic Editor: Young-Wook Kim \\ Copyright (C 2013 Jon C. Goldsby. This is an open access article distributed under the Creative Commons Attribution License, \\ which permits unrestricted use, distribution, and reproduction in any medium, provided the original work is properly cited. \\ Computational material methods were used to predict and investigate electrical and structural properties of cerium oxide $\left(\mathrm{CeO}_{2}\right)$. \\ Density functional theory was used to obtain the optimized crystal structure and simulate the material's electronic and elastic \\ responses. Oxygen to oxygen nearest neighbor distance is $2.628 \AA$, while oxygen to cerium distance is calculated to be $2.276 \AA$. The \\ conduction band has a prominent set of bands, which exists between 6 and $17 \mathrm{eV}$. An indirect energy gap (6.04 eV) exists between \\ the valence and conduction bands. The independent elastic constants allow a mechanical assessment on the suitability of cubic \\ cerium oxide as a substrate for advanced electronic devices. The calculated results of phonon dispersion curves are also given.
}

\section{Introduction}

A significant need exists to develop materials not only capable of providing desired electronic properties but also capable of surviving at extreme temperatures during device fabrication, such as in cofiring. An excellent candidate material is the oxide-based ceramic ceria. Ceria with its cubic structure can provide a platform for the introduction of dopants that can alter its electrical properties from insulating to semiconducting though oxygen vacancies [1-4]. This oxide-based ceramic material also possesses the refractory nature needed for high-temperature operation. However, the proper selection of dopants has to this point been somewhat speculative in nature based upon empirical experimentation. With the use of computational methods, material properties can be evaluated and optimized before the material is processed allowing an optimized structure to be realized quickly. This saves enormous time and efforts of experimentally assessing properties of multiple changes in composition and structure. The purpose of this investigation is to predict electronic and static and dynamic lattice response of pure ceria.

\section{Computational Methods}

The commercial software package Material Studio, including the Cambridge Serial Total Energy Package CASTEP module was used to conduct these simulations [5]. The calculations were performed on a parallel computer cluster consisting of 14 AMD Opteron 64-bit processors utilizing a LINUXbased operating system. Starting with the base structure, a geometric optimization was performed to determine the initial lattice parameters and density. In this calculation, the generalized gradient approximation (GGA) [6] was used along with ultrasoft pseudopotentials to represent the atoms. Specifically, for this geometric optimization, the PerdewBurke-Ernzerhof potential for solids (PBEsol) was used [7]. To obtain accurate band structures, calculations using the GGA PBEsol, as well as the hybrid functional Becke, threeparameter, Lee-Yang-Parr (B3LYP) potentials, were used $[8,9]$. The finite element displacement method applied to a supercell of the geometrically optimized primitive lattice using norm-conserving potentials [10] to calculate the phonon dispersion curves was used. The real space cutoff radius was set to $5 \AA$ which resulted in a supercell volume 27 


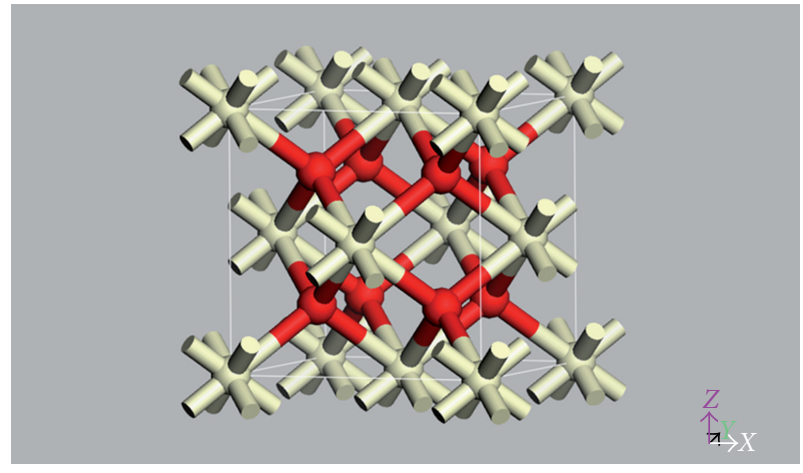

FIgURE 1: Cerium oxide Fm-3m oxygen atoms denoted in red.

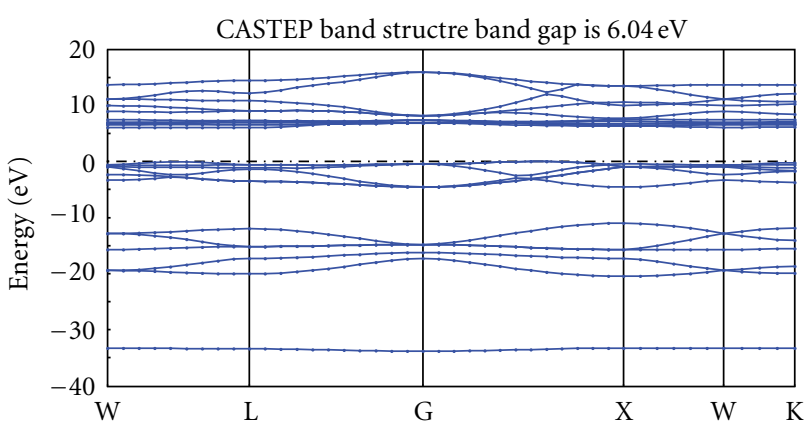

(a)

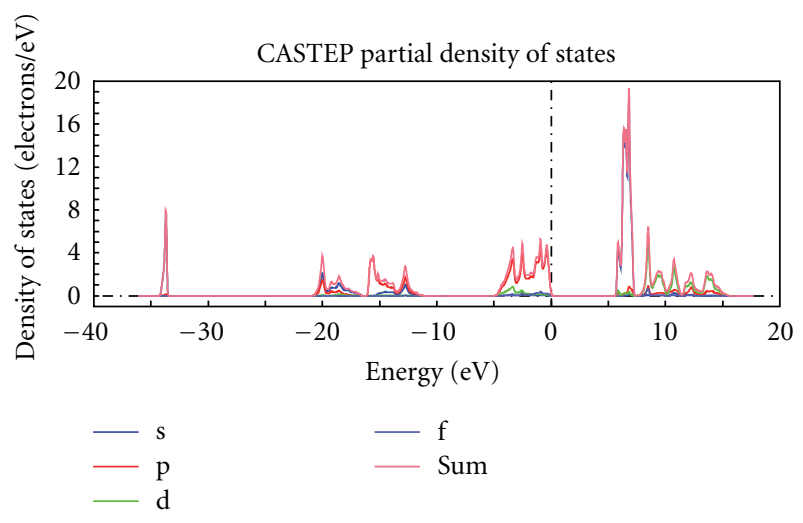

(b)

FIGURE 2: Electronic distribution in cubic ceria: band structure (a) and density of electron states (b).

times larger than the original unit cell. The quality of the qfactor spacing was set to $0.015 \AA^{-1}$.

\section{Results and Discussion}

3.1. Crystal Structure. The ceria structure used for these calculations possesses the $\mathrm{Fm}-3 \mathrm{~m}$ space group as seen in Figure 1. The geometric and electronic band structure calculations do not include thermal entropy effects. Therefore, these results are interpreted to occur at absolute zero temperature. The results of the calculation yielded a lattice parameter of $5.256 \AA$ compared to their room temperature expected value

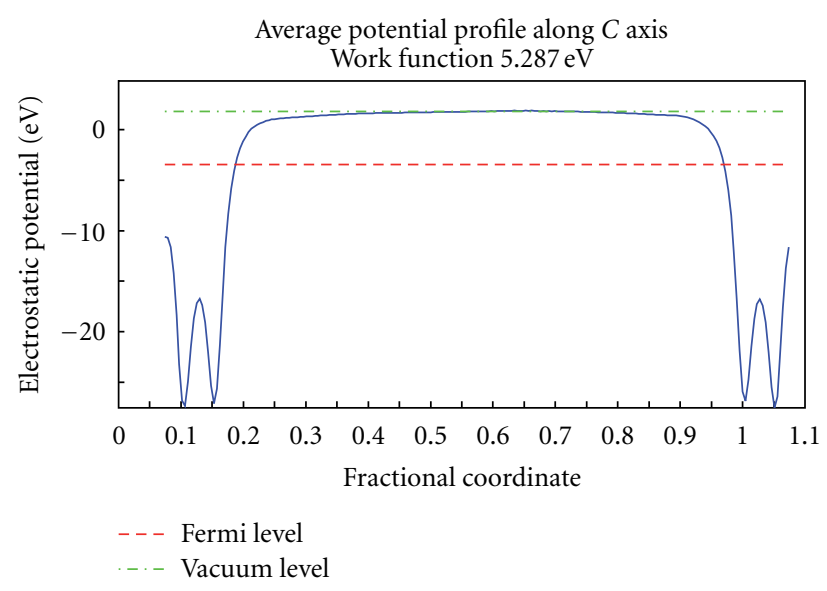

FIGURE 3: Distribution of the electrostatic potential in a vacuum between two (110) planes.

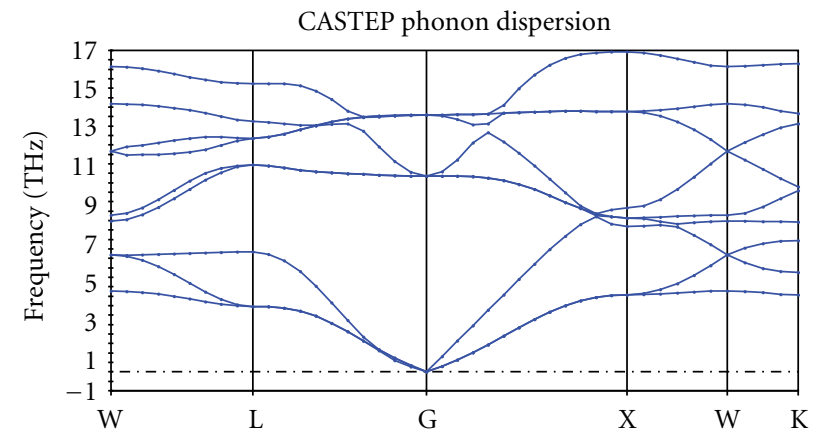

FIgURE 4: Phonon dispersion curves of cubic ceria.

of $5.410 \AA$ [11]. The cerium to cerium distance is $3.717 \AA$ while the next nearest neighbor cerium to cerium distance corresponds to the lattice parameter. The oxygen to oxygen nearest neighbor distance is $2.628 \AA$ while oxygen to cerium distance was calculated to be $2.276 \AA$. All lattice parameters remained orthogonal with the angles all being $90^{\circ}$. The total volume is $145.260 \AA^{3}$ with an overall density of $7.870 \mathrm{~g} / \mathrm{cm}^{3}$.

3.2. Electronic Properties. The starting electronic configurations are $\mathrm{Ce}[\mathrm{Xe}] 4 \mathrm{f}^{1} 5 \mathrm{~d}^{1} 6 \mathrm{~s}^{2}$ and $\mathrm{O}[\mathrm{He}] 2 \mathrm{~s}^{2} 2 \mathrm{p}^{4}$. Figure 2 shows the band structure for a standard ceria unit cell. Three major band groups below the Fermi level are found centered at the following positions at $-34,-15$, and $-2 \mathrm{eV}$, respectively, while other major band groups are located at 4 and $10 \mathrm{eV}$ above the Fermi level. The indirect band gap in the G-X direction has a value of $6.04 \mathrm{eV}$ which is in agreement with the experimental value [12]. The valence band begins at -5 to $-1 \mathrm{eV}$ and is dominated by the $\mathrm{p}$ orbital associated with the oxygen atoms. The lower band structures reveal large effective masses, due to the high radius of curvature of the bands, thereby prohibiting the promotion of carriers from below the Fermi level. The valence band consists of a spdf hybrid with dominant $\mathrm{p}$ character. In addition, the density of states for these bands is relatively small at $5 \mathrm{eV}$ per volt. At levels below $-10 \mathrm{eV}$ the charges are bound, consisting of 


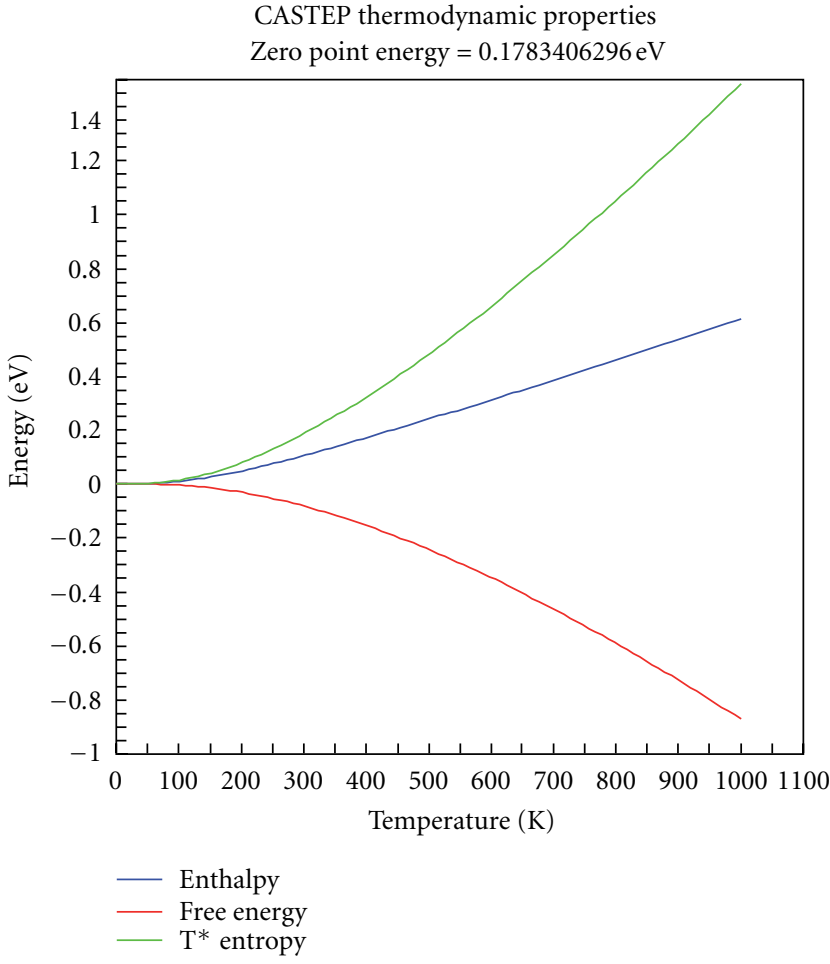

(a)

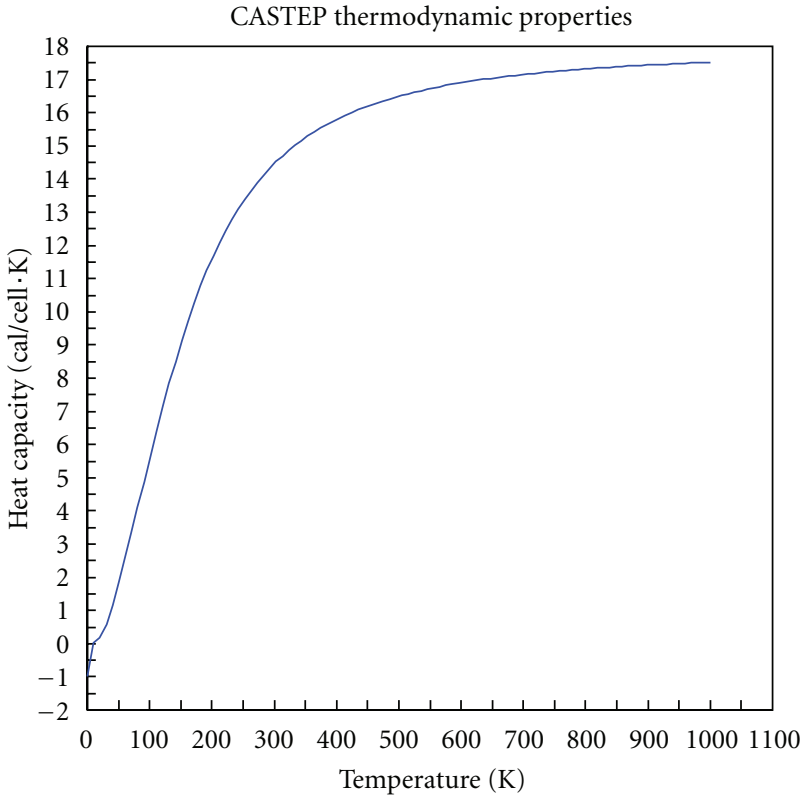

(b)

FIGURE 5: Thermodynamic properties enthalpy, free energy, and entropy (a) and heat capacity (b).

hybridized d, s, and p orbitals. The conduction band has a prominent set of bands which exists between 6 and $17 \mathrm{eV}$. The highest valence band occurs in the GX direction with a value of $17 \mathrm{eV}$. An oxygen p orbital beyond the Fermi level, centered at $10 \mathrm{eV}$, forms the bond with the cerium pdf hybrid. The cerium atom contributes to the majority of the conduction band dominated by an $f$ orbital and to a lesser degree $p$ as well as $\mathrm{d}$, which forms the oxygen bonds. However, the prominent $\mathrm{f}$ orbital above the Fermi level is reported to be unoccupied [13] but nonetheless forms a hybrid with some minor $p$ and $\mathrm{d}$ character. The work function was determined by constructing dual two-dimensional arrays of the $\mathrm{CeO} 2$ (110) surfaces separated by a vacuum gap of $30 \AA$. The electrostatic potential, determined midway of this gap, represents the energy required to remove one electron from the surface [14]. The (110) plane was selected as an intermediate between the (100) and (111) with regard to atomic population within a given plane. Figure 3 illustrates this potential profile between the two (110) planes in a vacuum. From this electrostatic potential versus fractional distance, the work function was calculated to be $5.287 \mathrm{eV}$.

3.3. Elastic Properties. The calculated elastic constants (Cij) elements are given in (1). From the three independent elastic constants $c_{11}, c_{12}$, and $c_{14}$ and bulk modulus $(B=277.521 \pm$ 0.348 (GPa)), the mechanical stability criteria of the cubic crystal may be evaluated using various symmetry-based relationships $[15,16]$. For this case, $c_{11}$ and $c_{44}$ must be greater than zero as well as the respective quantities $\left(c_{11}-c_{12}\right)$ and $\left(c_{11}+2 c_{12}\right)$. In addition, the bulk modulus must be greater than $c_{12}$ but less than $c_{11}$, thus for ceria the mechanical stability requirements are met:

$$
\begin{aligned}
& c i j[\mathrm{GPa}] \\
& \quad=\left[\begin{array}{cccclll}
455.06 & 188.74 & 188.74 & 0.00 & 0.00 & 0.00 \\
188.74 & 455.06 & 188.74 & 0.00 & 0.00 & 0.00 \\
188.74 & 188.74 & 455.06 & 0.00 & 0.00 & 0.00 \\
0.00 & 188.74 & 0.00 & 81.48 & 0.00 & 0.00 \\
0.00 & 188.74 & 0.00 & 0.00 & 81.48 & 0.00 \\
0.00 & 188.74 & 0.00 & 0.00 & 0.00 & 81.48
\end{array}\right]
\end{aligned}
$$

For single-crystal ceria the predicted Young's modulus is $344.39 \mathrm{GPa}$ along the three orthogonal axes. The polycrystalline bulk modulus was calculated as $277.52 \mathrm{GPa}$ using the Voigt model which is comparable to the experimental value of $220 \mathrm{GPa}$ [17]. The Poisson's ratio is 0.293 , and the shear modulus was calculated to be $102.15 \mathrm{GPa}$. The elastic anisotropic factor ratio was calculated to be 0.773 , which means polycrystalline ceria should be less susceptible to propagating microcracks at the grain boundary triple points [18], thereby facilitating its use as a suitable substrate for electronic devices.

3.4. Phonon Dispersion. The phonon is defined as the quantum of elastic strain energy. Therefore, the dispersion curves give insight into the material's thermal properties. For the cubic unit cell $(\mathrm{N})$, the maximum number of branches, based 
upon 3 atoms per cell ( $\mathrm{z}$ ), yields 9 degrees of freedom through the relationship $3 \mathrm{Nz}$ [19]. The relationship $(3 \mathrm{z}-3)$ yields a maximum of six optical branches and hence the balance of three acoustic branches in any given direction. Figure 4 shows results of the phonon dispersion calculations. The lower acoustic branch in the G-X direction shows one acoustic longitudinal of higher energy than the transverse acoustic branch. The phonon dispersion curves become nondegenerate for all branches in the $\mathrm{X}-\mathrm{K}$ direction giving the expected nine branches for this cubic structure. The higher frequency optical branches are split at the G symmetry point indicating some ionic bonding character [19]. The phonon dispersion calculation allows additional thermodynamic properties to be determined. The maximum Debye temperature was found to be $643 \mathrm{~K}$. Figure 5(a) illustrates the enthalpy, free energy, and the temperature-scaled entropy as a function of temperature. The zero-point energy was found to be $0.178 \mathrm{eV}$. Figure 5(b) shows the temperature-dependent heat capacity per unit cell with a maximum value of 17 calories per Kelvin near the Debye temperature. The enthalpy was found to be $-1914 \mathrm{eV}$.

\section{Conclusions}

It has been demonstrated that first-principles methods allow predictions of material properties and behavior of ceria. Judicious selection of a hybrid functional is necessary for the correct description of the electronic band structure for this insulating oxide-based material. Both static and dynamic lattice responses can be predicted, and the results can be used to guide processing and device fabrication. In future investigations, first-principles methods will be applied to obtain property predictions as a function of dopants and other processing variables.

\section{References}

[1] R. van de Krol and H. L. Tuller, "Electroceramics-the role of interfaces," Solid State Ionics, vol. 150, no. 1-2, pp. 167-179, 2002.

[2] R. Ramamoorthy, P. K. Dutta, and S. A. Akbar, "Oxygen sensors: materials, methods, designs and applications," Journal of Materials Science, vol. 38, no. 21, pp. 4271-4282, 2003.

[3] M. Ozawa, "Role of cerium-zirconium mixed oxides as catalysts for car pollution: a short review," Journal of Alloys and Compounds, vol. 277, pp. 886-890, 1998.

[4] G. Eranna, B. C. Joshi, D. P. Runthala, and R. P. Gupta, "Oxide materials for development of integrated gas sensors-a comprehensive review," Critical Reviews in Solid State and Materials Sciences, vol. 29, no. 3-4, pp. 111-188, 2004.

[5] M. D. Segall, P. J. D. Lindan, M. J. Probert et al., "First-principles simulation: ideas, illustrations and the CASTEP code," Journal of Physics, vol. 14, no. 11, pp. 2717-2744, 2002.

[6] J. P. Perdew, K. Burke, and M. Ernzerhof, "Generalized gradient approximation made simple," Physical Review Letters, vol. 77, no. 18, pp. 3865-3868, 1996.

[7] J. P. Perdew, A. Ruzsinszky, G. I. Csonka et al., "Restoring the density-gradient expansion for exchange in solids and surfaces," Physical Review Letters, vol. 100, no. 13, Article ID 136406, 2008.
[8] J. Paier, M. Marsman, and G. Kresse, "Why does the B3LYP hybrid functional fail for metals?" Journal of Chemical Physics, vol. 127, no. 2, Article ID 024103, 2007.

[9] J. Robertson, K. Xiong, and S. J. Clark, "Band structure of functional oxides by screened exchange and the weighted density approximation," Physica Status Solidi B, vol. 243, no. 9, pp. 2054-2070, 2006.

[10] G. J. Ackland, M. C. Warren, and S. J. Clark, "Practical methods in ab initio lattice dynamics," Journal of Physics, vol. 9, no. 37, pp. 7861-7872, 1997.

[11] E. A. Kümmerle and G. Heger, "The Structures of C- $\mathrm{Ce}_{2} \mathrm{O}_{3+\delta}$, $\mathrm{Ce}_{7} \mathrm{O}_{12}$, and $\mathrm{Ce}_{11} \mathrm{O}_{20}$," Journal of Solid State Chemistry, vol. 147, no. 2, pp. 485-500, 1999.

[12] E. Wuilloud, B. Delley, W. D. Schneider, and Y. Baer, "Spectroscopic evidence for localized and extended f-symmetry states in $\mathrm{CeO}_{2}$," Physical Review Letters, vol. 53, no. 2, pp. 202-205, 1984.

[13] D. R. Mullins, S. H. Overbury, and D. R. Huntley, "Electron spectroscopy of single crystal and polycrystalline cerium oxide surfaces," Surface Science, vol. 409, no. 2, pp. 307-319, 1998.

[14] C. W. Chen and M. H. Lee, "Ab initio calculations of dimensional and adsorbate effects on the workfunction of singlewalled carbon nanotube," Diamond and Related Materials, vol. 12, no. 3-7, pp. 565-571, 2003.

[15] D. M. Han, X. J. Liu, S. H. Lv, H. P. Li, and J. A. Meng, "Elastic properties of cubic perovskite $\mathrm{BaRuO}_{3}$ from first-principles calculations," Physica B, vol. 405, no. 15, pp. 3117-3119, 2010.

[16] G. V. Sin'ko and N. A. Smirnov, "Ab initio calculations of elastic constants and thermodynamic properties of bcc, fcc, and hcp Al crystals under pressure," Journal of Physics, vol. 14, no. 29, pp. 6989-7005, 2002.

[17] L. Gerward, J. S. Olsen, L. Petit, G. Vaitheeswaran, V. Kanchana, and A. Svane, "Bulk modulus of $\mathrm{CeO}_{2}$ and $\mathrm{PrO}_{2}$-an experimental and theoretical study," Journal of Alloys and Compounds, vol. 400, no. 1-2, pp. 56-61, 2005.

[18] V. Tvergaard and J. W. Hutchinson, "Microcracking in ceramics induced by thermal-expansion or elastic-anisotropy," Journal of the American Ceramic Society, vol. 71, no. 3, pp. 157-166, 1988.

[19] G. Burns, Solid State Physics, Academic Press, San Diego, Calif, USA, 1985. 

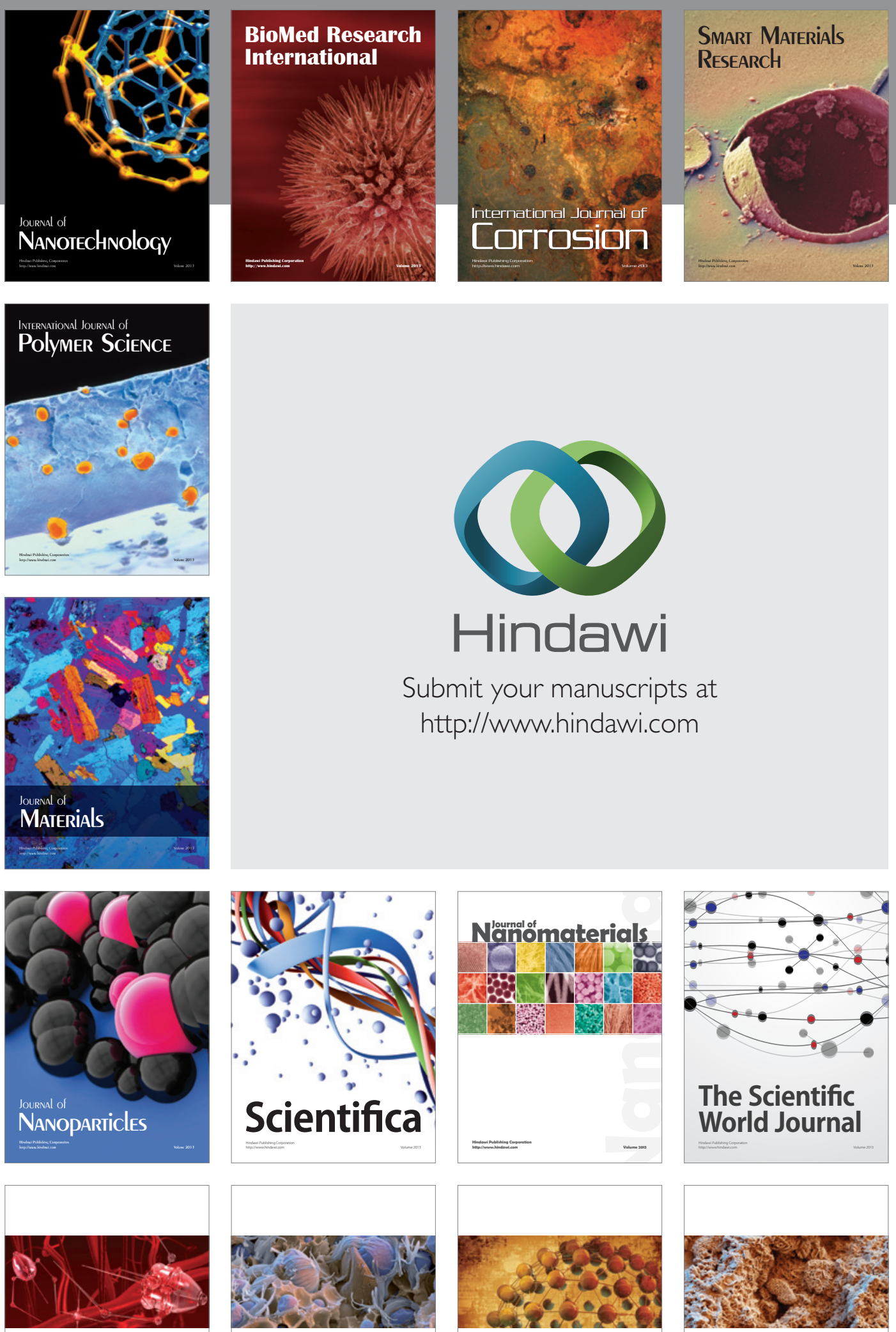

ISRN

Nanotechnology
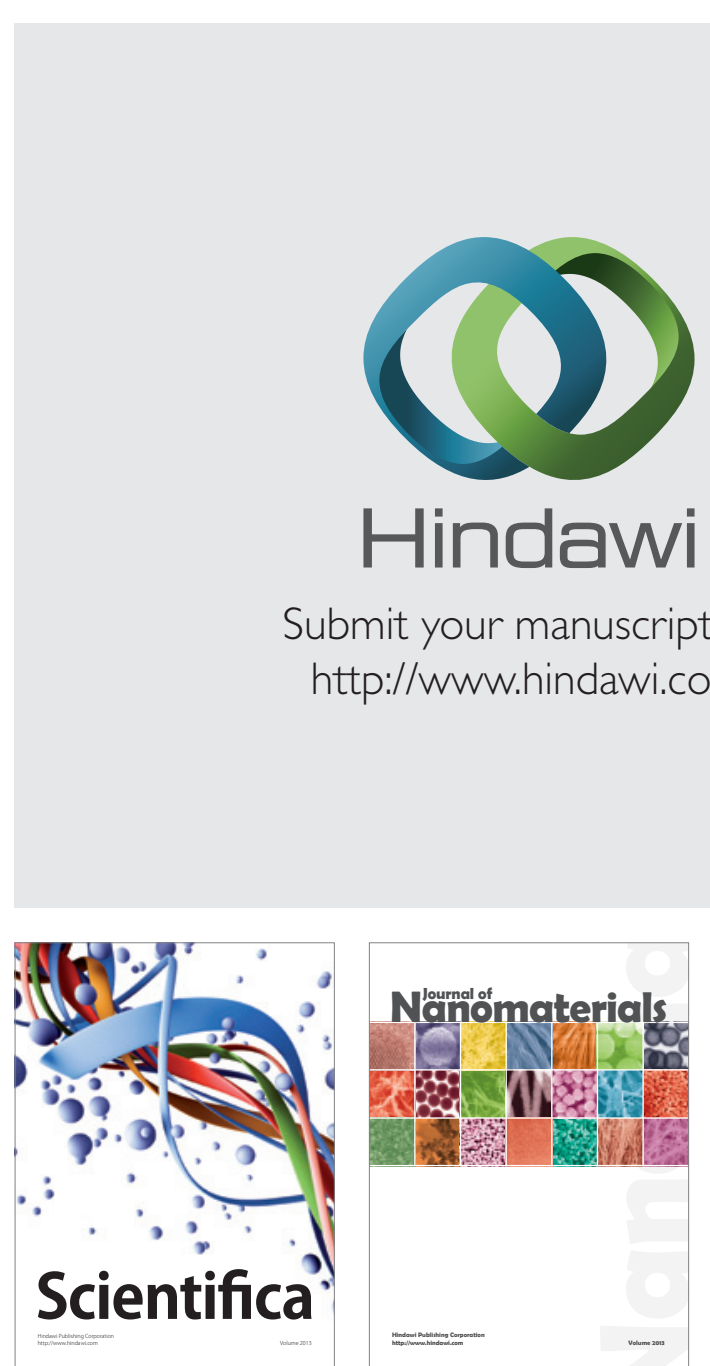

Submit your manuscripts at http://www.hindawi.com
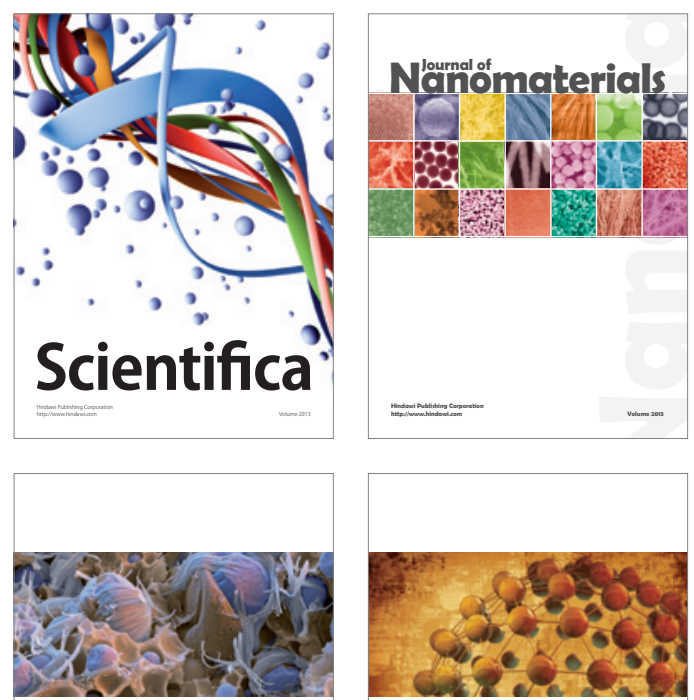

ISRN

Polymer Science

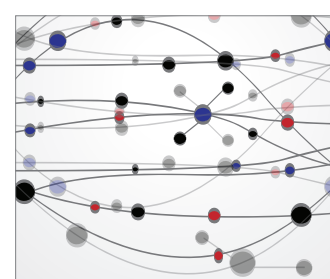

The Scientific World Journal

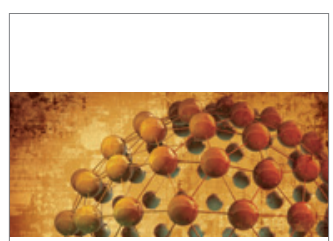

ISRN

Materials Science

\section{World Jounal}

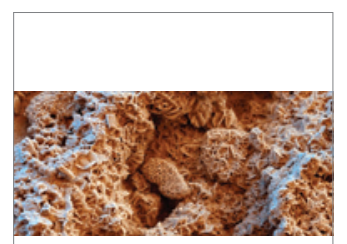

ISRN

Corrosion
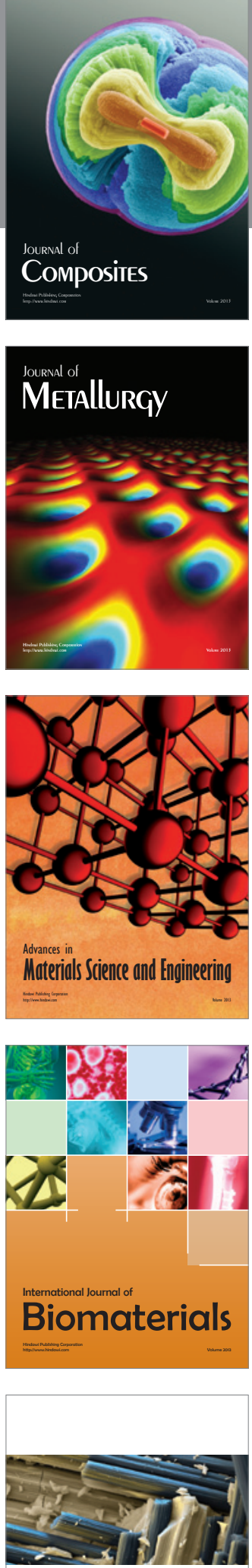

ISRN

Ceramics 